\title{
THE JACOBIAN MODULE OF A LIE ALGEBRA
}

\author{
J. P. BRENNAN, M. V. PINTO AND W. V. VASCONCELOS
}

\begin{abstract}
There is a natural way to associate to the commuting variety $C(A)$ of an algebra $A$ a module over a polynomial ring. It serves as a vehicle to study the arithmetical properties of $C(A)$, particularly Cohen-Macaulayness. The focus here is on Lie algebras and some of their representations.
\end{abstract}

\section{INTRODUCTION}

Let $V$ be a variety defined over an algebraically closed field $k$, equipped with a structure of $k$-algebra. The commuting variety, $\mathscr{C}(W)$, of a subvariety $W \subset V$, is the variety of commuting pairs of points of $W$. In the case that $V$ and $W$ are linear varieties, the coordinates of the commutator [ $x y]$ of two generic elements of $W$ provide an obvious set of equations for $\mathscr{C}(W)$.

Our aim here is to analyze this set of equations with regard to three questions: (i) Whether it gives the ideal of definition of $\mathscr{C}(W)$; (ii) to describe the ring of regular functions on $\mathscr{C}(W)$; and (iii) to study depth properties of this variety, in particular whether it is Cohen-Macaulay.

The answers will be shown to depend on an analysis of the fibers of the canonical projection $\mathscr{C}(W) \rightarrow W$. We introduce a module $E$ over the affine ring of $W$-to be called the Jacobian module of $\mathscr{C}(W)$-that plays an important role in capturing its reduced ideal of definition. It is an ancestor of the module of relative differentials of the morphism.

In $\S \S 1$ and 2 , we define $E$, derive some of its elementary properties-e.g. it may have any projective dimension-and give a mechanism for translating facts about the dimension of the components of $\mathscr{C}(W)$ into ideal theoretic data on the matrix of presentation of $E$ (Theorem 2.5). In the next section, as an application, we find the reduced equations for the commuting variety of the space of symmetric matrices along with other associated varieties, and prove they are Cohen-Macaulay (Theorem 3.1).

With the emphasis shifting to commuting varieties of Lie algebras, we study in $\S 4$ semisimple Lie algebras. A description of the ring of regular functions

Received by the editors September 2, 1988.

1980 Mathematics Subject Classification (1985 Revision). Primary 13H10; Secondary 13-04, 13C05, $13 \mathrm{C} 15$.

The second author was partially supported by CNPq, Brazil.

The third author was partially supported by the NSF. 
of their commuting varieties is given in Theorem 4.4, based on the so-called generic Cartan subalgebras.

The last section contains the construction of the generic Cartan subalgebras for the classical Lie algebras (and partly for algebras of type $G_{2}$ ), and as a consequence it is shown that $E$ has projective dimension two (Theorem 5.1). It exploits the classification theory of such algebras but still leaves out most of the exceptional algebras. Large-scale computer experiments suggest that these commuting varieties are always Cohen-Macaulay.

We would like to thank J. Herzog and S. Washburn for many helpful and stimulating discussions.

\section{The Jacobian module}

This section introduces the Jacobian module attached to a commuting variety. It is focused on linear varieties. Throughout $k$ is an algebraically closed field, of characteristic zero. This is not strictly required since we could, often, get away with fields of characteristic different from two. It will make however for a uniform setting. For basic terminology and facts of commutative algebra, we shall use [15].

Let $V$ be a linear variety with an algebra structure and let $W$ be one of its linear subvarieties. Let $\left\{e_{1}, e_{2}, \ldots, e_{n}\right\}$ and $\left\{f_{1}, f_{2}, \ldots, f_{m}\right\}$ be bases of $W$ and $V$, and consider two independent generic elements of $W: x=\sum x_{i} e_{i}$ and $y=\sum y_{i} e_{i}$. Denote by $[x y]$ the commutator of $x$ and $y$. When written out in terms of the basis of $V$ the coefficients are forms of degree two that generate a defining ideal $J(W)$ for the commuting variety of $W, \mathscr{C}_{V}(W)=\mathscr{C}(W)$, in the affine space $k^{2 n}$. It is clear that this ideal does not depend on the choices of bases.

Denote by $\varphi$ the Jacobian submatrix of $J(W)$ with respect to the subset $\left\{y_{1}, y_{2}, \ldots, y_{n}\right\} . \varphi$ is a matrix of linear forms of the ring

$$
R=k\left[x_{1}, x_{2}, \ldots, x_{n}\right] \text {. }
$$

Definition 1.1. The Jacobian module of the commuting variety of $W$ is the $R$-module $E=\operatorname{cokernel}(\varphi)$.

Whenever $W=V$, by abuse of terminology, we call $E$ the Jacobian module of $V$. In this case there is a more direct description of the Jacobian module. Let $\left\{e_{1}, \ldots, e_{n}\right\}$ be a basis of $V$, and denote by $R$ the ring of regular functions on $V, R=k\left[x_{1}, \ldots, x_{n}\right]$. Put $x=\sum x_{i} e_{i}$. The exact sequence

$$
V \otimes R \stackrel{(\operatorname{ad} x)^{t}}{\longrightarrow} V \otimes R \rightarrow E \rightarrow 0
$$

ad $x(a)=[a x]$ defines the Jacobian module.

To show the significance of $E$ we recall the definition of the symmetric algebra of a module. Let $R$ be a Noetherian ring and $E$ a finitely generated $R$-module. When $E$ is given by the presentation

$$
R^{m} \stackrel{\varphi}{\rightarrow} R^{n} \rightarrow E \rightarrow 0, \quad \varphi=\left(a_{i j}\right)
$$


its symmetric algebra $S(E)$ is simply the quotient of the polynomial ring $R\left[T_{1}, \ldots, T_{n}\right]$ by the ideal $J(E)$ generated by the 1 -forms in the $T_{i}$ 's-variables

$$
f_{j}=a_{1 j} T_{1}+\cdots+a_{n j} T_{n}, \quad j=1, \ldots, m .
$$

The following identification is now obvious:

Proposition 1.2. $\mathscr{C}(W) \cong \operatorname{Spec}(S(E))_{\text {red }}$.

By way of illustration, let us consider

Example 1.3. (a) Let $L$ be the 3-dimensional Lie algebra $\{e, f, g\}$ defined by

$$
[e f]=0, \quad[e g]=e, \quad[f g]=f .
$$

$W=V$ is affine 3-space. The ideal $J(W)$ is defined by the forms $x_{1} y_{3}-x_{3} y_{1}$ and $x_{2} y_{3}-x_{3} y_{2}$. The Jacobian module $E$ has a presentation

$$
\begin{gathered}
0 \longrightarrow R^{2} \stackrel{\varphi}{\longrightarrow} R^{3} \longrightarrow E \longrightarrow 0, \\
\varphi=\left(\begin{array}{cc}
-x_{3} & 0 \\
0 & -x_{3} \\
x_{1} & x_{2}
\end{array}\right) .
\end{gathered}
$$

It is easy to see that $S(E)$ is reduced, so that $\mathscr{C}(W)=\operatorname{Spec}(S(E))$. It has two irreducible components.

(b) Denote by $D S_{n}$ the space of all $n \times n$ square matrices with equal line sums - that is, essentially doubly stochastic matrices. If $n=3$, a calculation with the Bayer and Stillman Macaulay program shows that the Jacobian module $E$ of $\mathscr{C}\left(D S_{3}\right)$ has projective dimension two and $S(E)$ is a Cohen-Macaulay integral domain. (We do not know what happens for arbitrary $n$.)

(c) Let $V$ be an $n$-dimensional vector space over $k$. There is a natural Lie algebra structure on $L=V \oplus \bigwedge^{2} V$ that makes $\wedge^{2} V$ the center of $L$. The Jacobian module of $L$ is the direct sum of a free module of rank $n(n-1) / 2$, corresponding to its center, and the module that has $n$ generators and for relations the forms $x_{i} y_{j}-x_{j} y_{i}, 1 \leq i<j \leq n$-that is, the ideal $\left(x_{1}, \ldots, x_{n}\right)$. The projective dimension of Jacobian modules can thus attain any value.

(d) Finally, let $H_{n}$ be the Heisenberg algebra of dimension $2 n+1$. The commuting variety $\mathscr{C}\left(H_{n}\right)$ is defined by a single equation

$$
\sum_{i=1}^{i=n} x_{i} y_{n+i}-x_{n+i} y_{i} \text {. }
$$

It is a factorial variety for $n \geq 2$.

There are also examples showing $S(E)$ with components of different dimensions.

Remark. The connection between the Jacobian module of $\mathscr{C}(W)$ and its module of differentials is the following. Let $B$ be the affine ring of $\mathscr{C}(W)$, 
$B=C / I, C=R\left[y_{1}, \ldots, y_{n}\right]$. The module of relative differentials $\Omega_{B / R}$ is given by the exact sequence

$$
I / I^{2} \stackrel{d}{\longrightarrow} \Omega_{C / R} \otimes B \longrightarrow \Omega_{B / R} \longrightarrow 0 .
$$

Since $J(W) \subset I$, there exists a canonical surjection

$$
E \otimes B \rightarrow \Omega_{B / R},
$$

which is another way of expressing the fact that the set of equations in $J(W)$ provides an approximation of $I$.

\section{THE FIBERS OF THE COMMUTING VARIETY}

We shall now begin to show the naturality of the Jacobian module of $\mathscr{C}(W)$ by proving a result that translates information about its irreducible components into ideal theoretic data on the determinantal ideals of the matrix $\varphi$. For this end, we recall how the dimension of a symmetric algebra may be calculated. It appeals to a reformulation in [22] of a result of [11].

The following notion plays an important role in the analysis of the fibers of the canonical morphism $\operatorname{Spec}(S(E)) \rightarrow \operatorname{Spec}(R)$ (cf. [11, 22]). Let $R$ be a Noetherian domain and let $\varphi: R^{m} \rightarrow R^{n}$ be a presentation of the $R$-module $E$. More generally, $R$ could be a reduced ring and $E$ an $R$-module with a rank, that is for each minimal prime $P, E_{P}$ is a free $R_{P}$-module of constant rank. For each integer $t \geq 1$ denote by $I_{t}(\varphi)$ the ideal generated by the $t \times t$ minors of $\varphi$. For a nonnegative integer $k$, the condition $\mathscr{F}_{k}$ is defined by the sliding requirements on the sizes of the $I_{t}(\varphi)$ 's (cf. $\left.[9,22]\right)$ :

$$
\text { height } I_{t}(\varphi) \geq \operatorname{rank}(\varphi)-t+1+k, \quad 1 \leq t \leq \operatorname{rank}(\varphi) .
$$

The condition $\mathscr{F}_{0}$-or rather, how deeply it is violated-reads the Krull dimension of $S(E)$. To be precise, set $m_{0}=\operatorname{rank}(\varphi)$, so that $\operatorname{rank}(E)=n-m_{0}$. Define the following measure on $[1, \operatorname{rank}(\varphi)]$ :

$$
d(t)=\left\{\begin{array}{l}
m_{0}-t+1-\text { height } I_{t}(\varphi) \quad \text { if } \mathscr{F}_{0} \text { fails at } t, \\
0 \quad \text { otherwise } .
\end{array}\right.
$$

Put $d(E)=\sup _{t}\{d(t) \mid 1 \leq t \leq \operatorname{rank}(\varphi)\}$; one has the following dimension formula (see [22]).

Theorem 2.1. Let $R$ be a reduced equidimensional catenarian ring and let $E$ be a finitely generated $R$-module with a rank. Then the Krull dimension of $S(E)$ is $\operatorname{dim} R+\operatorname{rank} E+d(E)$.

It follows that if the Krull dimension of $S(E)$ has the expected value, $\operatorname{dim} R+\operatorname{rank} E$, then $\mathscr{F}_{0}$ is satisfied. The case that we are interested in is:

Proposition 2.2. Let $E$ be a finitely generated graded module over $R=$ $k\left[x_{1}, \ldots, x_{n}\right]$. Then $\operatorname{Spec}(S(E))$ is irreducible if and only if $E$ satisfies $\mathscr{F}_{1}$ and all the minimal prime ideals of $S(E)$ have the same dimension.

Proof. One of the minimal prime ideals of a symmetric algebra $S(E)$ is the $R$-torsion submodule $T$ of $S(E)$. Since the Krull dimension of $S(E) / T$ is 
$\operatorname{dim} R+\operatorname{rank} E$ (cf. [11,22]), it follows that if $S(E)$ is equidimensional then by Theorem 2.1 the condition $\mathscr{F}_{0}$ is automatically satisfied. Thus both conditions in the assertion imply $\mathscr{F}_{0}$, and that $E$ is free in codimension one. In particular, for any nonunit $x$ of $R \operatorname{rank}_{R /(x)}(E / x E)=\operatorname{rank}_{R}(E)$.

Suppose $\operatorname{Spec}(S(E))$ is irreducible; then for each nonzero element $x$ of $R$, as $x \notin T, \operatorname{dim} S(E) \otimes R /(x)=\operatorname{dim} S(E)-1$. But $S_{R}(E) \otimes R /(x) \cong$ $S_{R /(x)}(E / x E)$, so that the algebra $S(E / x E)$ will satisfy the condition of Theorem 2.1 if $R /(x)$ is reduced. It is not difficult to see that we can pick a square-free element $x$ contained in all the associated primes of the $I_{t}(\varphi)^{\prime} s$. Then $\mathscr{F}_{1}$ will follow.

Conversely, suppose $\mathscr{F}_{1}$ holds and $M$ is a minimal prime of $S(E)$ other than $T$. According to [11] $M$ has the following description: If $P=M \cap R$, then $M$ is the inverse image in $S(E)$ of the $R / P$-torsion submodule of $S_{R / P}(E / P E)$. If however $P \neq 0$, reducing $E$ modulo a prime element $x$ of $P$ would, in the presence of $\mathscr{F}_{1}$, yield an $R /(x)$-module $E / x E$ whose $R /(x)$-rank is still rank $E$, so that the dimension of $S(E / x E)$, by Theorem 2.1 , is one less than that of $S(E)$. The equidimensionality hypothesis on $S(E)$ rules this out.

There are some immediate consequences for commuting varieties.

Corollary 2.3. If $\mathscr{C}(W)$ is Cohen-Macaulay, and its Jacobian module satisfies $\mathscr{F}_{1}$ then $\mathscr{C}(W)$ is irreducible.

The condition that $\operatorname{Spec}(S(E))$ be irreducible does not suffice to ensure it is also reduced. On the affirmative side, one has (see [1, 9, 10, 21, Theorem 3.4]):

Theorem 2.4. Let $R$ be a Cohen-Macaulay domain and let $E$ be a finitely generated $R$-module of projective dimension one. Then $S(E)$ is an integral domain if and only if $E$ satisfies $\mathscr{F}_{1}$. In this case $S(E)$ is a local complete intersection (over $R$ ).

We embed this discussion into the following basic result:

Theorem 2.5. If the commuting variety $\mathscr{C}(W)$ is irreducible, then

(1) height $I_{t}(\varphi) \geq \operatorname{rank}(\varphi)-t+2$, for $1 \leq t \leq \operatorname{rank}(\varphi)$.

(2) If $E$ has projective dimension 1 then $S(E)$ is a domain.

(3) If $E$ has projective dimension 2 then $E$ is torsion-free.

Proof. The first two parts having been proven above, we turn to the proof of (iii).

Let

$$
0 \rightarrow R^{l} \rightarrow R^{m} \stackrel{\varphi}{\rightarrow} R^{n} \rightarrow E \rightarrow 0
$$

be a projective resolution of $E$. If $P$ is an associated prime ideal of $E$, it must have height at most two (cf. [15, Theorem 19.1]). We claim that $P=(0)$.

Denoting still by $R$ the localization at $P$, we assume that the resolution above is minimal. Since $E$ satisfies the condition $\mathscr{F}_{1}$, height $I_{1}(\varphi) \geq(m-l)-$ $1+2$; thus if $E$ is not free, height $P=2$ and $m-l=1$. This means that 
there is an exact sequence

$$
0 \rightarrow I \rightarrow R^{r} \rightarrow E \rightarrow 0
$$

where $I$ is a rank one, nonfree module and $E$ is free in codimension one; $I$ may be identified to an ideal of height two. We claim that the symmetric algebra $S(E)$ has at least 2 minimal primes. If $I=\left(a_{1}, \ldots, a_{s}\right)$, denote by $f_{i}$ the image of $a_{i}$ in $R^{r}=R T_{1} \oplus \cdots \oplus R T_{r}$. The symmetric algebra of $E$ is $R\left[T_{1}, \ldots, T_{r}\right] /\left(f_{1}, \ldots, f_{s}\right)$. Since height $(I)=2$, for any $a_{i}$ there exists an element $b_{i} \in I$ such that $\left\{a_{i}, b_{i}\right\}$ is a regular sequence. If we denote by $g_{i}$ the image of $b_{i}$, we must have $a_{i} g_{i}=b_{i} f_{i}$ since they are both the image of the element $a_{i} b_{i}$. This implies that $f_{i}$ must be a multiple of $a_{i}$. Thus $\left(f_{1}, \ldots, f_{s}\right)=(I f)$, for some 1-form $f$.

It follows that if $E$ has projective dimension two, then all forms linear in the sets of $x$ or $y$ variables in the ideal of definition of $\mathscr{C}(W)$ have been accounted for-in particular all quadrics and cubics.

\section{SYMMETRIC MATRICES}

Let $k$ be an algebraically closed field, of characteristic 0 , and let $S_{n}(k)$ be the affine space of all symmetric matrices of order $n$ with entries in $k$. The commuting variety of $S_{n}(k)$ is defined by the ideal generated by the entries of

$$
Z=[X, Y]=X \cdot Y-Y \cdot X,
$$

where $X$ and $Y$ are generic symmetric $n \times n$ matrices in $n(n+1)$ indeterminates. $Z=\left[z_{i j}\right]$ is an alternating matrix of 2 -forms.

The main application of this section is

Theorem 3.1. The entries of $Z$ form a regular sequence generating a prime ideal.

The proof will follow from Theorem 2.5, once certain details of the structure of the Jacobian module are made clear.

Lemma 3.2. The Jacobian module of $\mathscr{C}\left(S_{n}(k)\right)$ has projective dimension one.

Proof. It suffices to show that the presentation matrix $\varphi$ of $E$ has rank $n(n-1) / 2$. If we specialize the matrix $X$ to a generic diagonal matrix, it is easy to see that the forms $z_{i j}$ specialize to

$$
z_{i j}^{*}=\left(x_{i i}-x_{j j}\right) y_{i j}
$$

so that the corresponding matrix has full rank.

The next result is a key geometric ingredient. It was pointed out to us, independently, by Robert Guralnick and David Rohrlich.

Proposition 3.3. $\mathscr{C}\left(S_{n}(k)\right)$ is an irreducible variety.

Proof. It suffices to show that if $W$ is a generic symmetric matrix commuting with $X$ then the pair $(X, W)$ is a generic point of $\mathscr{C}\left(S_{n}(k)\right)$. This is a formal 
consequence of the proof of [6, Theorem 1, pp. 341-342] once Lemmas 3.4 and 3.5 have been established.

We recall that a square matrix is nonderogatory provided its minimal polynomial is its characteristic polynomial.

Lemma 3.4. Let $A$ be a square matrix. Then the following are equivalent.

(1) $A$ is nonderogatory.

(2) If $B$ is a matrix and $[A, B]=0$, then there is a polynomial $p(t)$ with $p(A)=B$.

Proof. This is [6, Proposition 4].

Lemma 3.5. Let $B$ be an element of $S_{n}(k)$. There exists a nonderogatory element of $S_{n}(k)$ that commutes with $B$.

Proof. By [5, Corollary 2, p. 13] and the Jordan decomposition theorem, there exists an orthogonal matrix $O$ such that

$$
O^{t} B O=\bigoplus_{i=1}^{s}\left(\lambda_{i} I_{i}+N_{i}\right),
$$

with $N_{i}$ nilpotent, symmetric, and $\lambda_{i} I_{i}+N_{i}$ irreducible. ( $M^{t}$ is the transpose of the matrix $M$.) The matrix

$$
O\left(\bigoplus_{i=1}^{s}\left(\mu_{i} I_{i}+N_{i}\right)\right) O^{t}
$$

with distinct $\mu_{i}$ 's, is nonderogatory and commutes with $B$.

Here is an application, in the manner of [7,8 and 18]. For each integer $0 \leq r \leq n$ define

$$
M_{n}^{r}=\left\{(A, B) \in S_{n}(k) \times S_{n}(k) \mid \operatorname{rank}[A, B] \leq r\right\} .
$$

Corollary 3.6. $M_{n}^{2 r}=M_{n}^{2 r+1}$ is an irreducible Gorenstein variety of codimension $(n-2 r-1)(n-2 r) / 2$. Its reduced equations are the Pfaffians of $Z$ of order $2 r+2$.

Proof. The entries of $Z=\left[z_{i j} \mid 1 \leq i<j \leq n\right]$, are homogeneous elements forming a regular sequence in $A=k\left[x_{p q}, y_{p q} \mid 1 \leq p \leq q \leq n\right]$, so that the inclusion $R=k\left[z_{i j}\right] \subset A$ is a faithfully flat homomorphism; see [15, p. 176]. Furthermore, by Theorem 3.1 the irrelevant maximal ideal of $R, \mathbf{p}=\left(z_{i j}\right)$, extends to a prime ideal of $A, P=\mathbf{p} A$.

Denote by $I$ the ideal of $R$ generated by the Pfaffians of order $2 \mathrm{~s}$ of the matrix $Z$. According to [14, Theorem 17], $I$ is a prime, Gorenstein ideal of $R$, with the codimension given by the formula above. In addition, the singular locus of this variety is given by the ideal of Pfaffians of order $2(s-1)$; in particular it is normal.

We now show that the homogeneous ideal $J=I A$ is prime. We claim that the zero divisors of $A / J$ all lie in $P$. Because the associated primes of $A / J$ 
are graded, we only have to check that for homogeneous polynomials $f \in A \backslash P$. By the graded version of [15, Theorem 22.5]

$$
0 \rightarrow A \stackrel{f}{\rightarrow} A \rightarrow A /(f) \rightarrow 0,
$$

is an exact sequence of flat $R$-modules, since $f$ is regular mod $\mathbf{p}$. Therefore multiplication by $f$ is also regular on any module $M \oplus_{R} A$.

To verify that $J$ is prime it suffices therefore to check primality in the localization $J_{P}$. We show that $J A_{P}$ is analytically irreducible. By going over to the completion $B$ of $A_{P}$ at the maximal ideal, the question reduces to verifying a local version of [14, Theorem $17(\mathrm{i})]$. Precisely, if $B=K\left[\left[z_{i j}\right]\right]$ ( $K$ is the appropriate coefficient field), as $I B$ is defined over $K\left[z_{i j}\right]$, the normality hypothesis sets up the conditions for an application of Zariski's theorem on analytical normality (cf. [17, Theorem 37.5]).

Remarks. 1. In contrast, in the case of arbitrary (or skew-symmetric) matrices one does not know the reduced equations for these varieties. The explanation seems to lie, as we shall discuss in the next section, in the more complicated structure of the Jacobian modules for these varieties.

2. Note that the proof above does not establish the normality of $M_{n}^{r}$; $\mathscr{C}\left(S_{n}(k)\right)=M_{n}^{0}$ itself is factorial for $n \leq 4$. To establish that $\mathscr{C}\left(S_{n}(k)\right)$ is factorial for all $n$, the condition $\mathscr{F}_{2}$ must hold for its Jacobian module $E$. This would be a consequence of the following geometric statement. Let $f$ be a nonzero form contained in the highest fitting ideal of the presentation matrix of $E$. Then the assertion of Lemma 3.5 remains valid for the hypersurface $(f=0)$ of $S_{n}(k)$.

3. Craig Huneke has pointed out to us that the use above of Zariski's theorem is not really needed, since homogeneous prime ideals are always analytically irreducible at the irrelevant ideal. The extra structure suggests that all these varieties are, at least, normal.

\section{Generic Cartan subalgebras}

From now on $L$ is a semisimple Lie algebra over an algebraically closed field $k$, of characteristic zero. Denote by $R$ the polynomial ring $k\left[x_{1}, \ldots, x_{n}\right]$, $n=\operatorname{dim} L$, and let $\mathscr{L}=L \otimes R$. The Jacobian module of $L$ is given by the presentation

$$
0 \rightarrow \mathscr{C} \rightarrow \mathscr{L} \stackrel{\varphi}{\rightarrow} \mathscr{L} \rightarrow E \rightarrow 0
$$

where $\varphi(a)=[x a]$.

In the Lie algebra $\mathscr{L} \otimes K, K=$ field of quotients of $R, x$ is a regular element, so that its centralizer $H$ is a Cartan subalgebra. Since the dimension of $H$ over $K$ is equal to the rank $l$ of $L$ and $\mathscr{C}=H \cap \mathscr{L}, \mathscr{C}$ is a finitely generated $R$-module of rank $l$.

Definition 4.1. $\mathscr{C}$ is the generic Cartan subalgebra of $L$.

To make use of this complex we must know considerably more about the mapping $\varphi$ and the algebra $\mathscr{C}$. Regarding the determinantal ideals of $\varphi$, 
the following result of Richardson [19] is crucial to our understanding of the Jacobian module of Lie algebras. (The case of $n \times n$ matrices was proved earlier by Motzkin and Taussky [16] and Gerstenhaber [6].)

Theorem 4.2. The commuting variety of a semisimple Lie algebra is irreducible.

We can apply Proposition 2.2 to obtain

Proposition 4.3. If $L$ is a semisimple Lie algebra, then its Jacobian module satisfies the condition $\mathscr{F}_{1}$.

There are two direct consequences of the semisimplicity of $L$ that we shall make use of. Denote by $B($,$) the Killing form of L$. If a basis $\left\{e_{1}, \ldots, e_{n}\right\}$ of $L$ is chosen so that $B\left(e_{i}, e_{j}\right)=\delta_{i j}$, it follows that the structure constants $c_{i j k}$ of $L$ satisfy the equation $c_{i j k}=c_{k i j}$. A simple calculation shows that in such basis the matrix $\varphi$ is skew-symmetric. This permits identifying the $R$-module $\mathscr{C}$ with the $R$-dual $E^{*}$ of $E$.

The other observation is that since $x$ is a semisimple element, $\operatorname{ker}(\varphi)=$ $\operatorname{ker}\left(\varphi^{2}\right)$, so that using the snake lemma on the composition of $\varphi$ with itself, we get the exact sequence

$$
0 \rightarrow \operatorname{ker}(\varphi) \rightarrow \operatorname{ker}\left(\varphi^{2}\right) \rightarrow \operatorname{ker}(\varphi) \rightarrow \operatorname{coker}(\varphi) \rightarrow \operatorname{coker}\left(\varphi^{2}\right) \rightarrow \operatorname{coker}(\varphi) \rightarrow 0
$$

and therefore

$$
0 \rightarrow \mathscr{C} \rightarrow E \rightarrow \operatorname{coker}\left(\varphi^{2}\right) \rightarrow E \rightarrow 0 .
$$

This yields a canonical map from the symmetric algebra of $\mathscr{C}$ into $S(E)$.

A set of generators for the affine ring of the commuting variety of $L$ can be obtained in the following manner. We are grateful to J. Herzog for several conversations on this matter.

Let $z_{1}, \ldots, z_{l}$ be a basis for the Cartan subalgebra of $L \otimes K$; pick the $z_{i}$ 's lying in $\mathscr{L}$. Let $T_{1}, \ldots, T_{l}$ be a set of $l$ new indeterminates. The symmetric algebra $S(E)=k[X, Y] / I_{1}([X Y])$ maps onto the subring $D$ of $R\left[T_{1}, \ldots, T_{l}\right]$ generated by coordinates of the element $z=\sum_{i=1}^{i=l} T_{i} z_{i}$ by specializing $Y \mapsto z$.

More precisely, write each $z_{i}=\sum_{j=1}^{j=n} b_{i j} e_{j}$ and consider the homomorphism

$$
\Phi: S(\mathscr{L}) \rightarrow R\left[T_{1}, \ldots, T_{l}\right], \quad e_{j} \mapsto \sum_{k=1}^{k=l} b_{k j} T_{k} .
$$

On $\left[e_{i} x\right]=\sum_{j=1}^{j=n} f_{i j} e_{j}$

$$
\Phi\left(\left[e_{i} x\right]\right)=\sum_{j=1}^{j=n} f_{i j} \Phi\left(e_{j}\right)=\sum_{k=1}^{k=l}\left(\sum_{j=1}^{j=n} f_{i j} b_{k j}\right) T_{l} .
$$

But

$$
\sum_{j=1}^{j=n} f_{i j} b_{k} j=B\left(\left[e_{i} x\right], x\right)=0 .
$$

Since $\Phi$ vanishes on the image of ad $(x)$, it induces the desired map from $S(E)$ to $D$. 
Theorem 4.4. $\mathscr{C}(L)=\operatorname{Spec}(D)$.

Proof. $D$ has obviously transcendence degree $\operatorname{dim} L+l$ over $k$. By Richardson's theorem and Proposition 1.2, the nilradical of $S(E)$ must be the kernel of the homomorphism.

$D$ has a more natural description when the $z_{i}$ generate all of $\mathscr{C}$. $\Phi$ restricted to $\mathscr{C}$ induces mappings

$$
S(\mathscr{C}) \hookrightarrow D \hookrightarrow R\left[T_{1}, \ldots, T_{\ell}\right]
$$

The composite is the mapping of symmetric algebras derived from the homomorphism of free modules $A: R^{l} \rightarrow R^{l}$ associated to the Cartan matrix $\left[B\left(z_{i}, z_{j}\right)\right]$. Note that none of these algebra homomorphisms is finite.

\section{Classical algebras}

The main result of this section is

Theorem 5.1. Let $L$ be a semisimple Lie algebra of type $A_{l}, B_{l}, C_{l}, D_{l}$ or $G_{2}$. Its generic Cartan subalgebra $\mathscr{C}$ is a free $R$-module. ${ }^{1}$

The proof appeals to the classification theorem (see $[2,12])$. In each instance we pick an appropriate representation. There must exist a single proof dealing with all algebras, rather than the case-by-case approach we are going to take. One advantage of this approach, however, is to obtain additional information on how $\mathscr{C}$ embeds into $\mathscr{L}$.

1. Algebras of type $A_{l}$. We may assume that $L=g l(l+1)$. In this and the other cases, the method we use is the following. First, to exhibit a free submodule $C_{0}$ of $C$ of rank $l$; then to show that the ideal generated by the minors of maximal order of the embedding $\psi: \mathscr{C}_{0} \rightarrow \mathscr{L}$ has codimension at least two; in other words, the embedding splits in codimension one. That this suffices follows from the acyclicity lemma (see [3]). $R$.

Here $\mathscr{C}_{0}$ is the subring $S=R[x]$ of $\mathscr{L}$, viewed as a matrix algebra over

Lemma 5.2. $S$ is regular in codimension tiwo.

Proof. $S$ is a complete intersection, $S=R[T] /(f(T))$, where $f(T)$ is the Cayley-Hamilton polynomial of the generic matrix $x$ :

$$
f(T)=T^{l+1}+\sigma_{1} T^{l}+\cdots+(-1)^{l+1} \sigma_{l+1}, \quad \sigma_{l+1}=\operatorname{det}(x) .
$$

Let us estimate the codimension of the Jacobian ideal of the polynomial $f(T)$ :

$$
J=\left(f(T), f^{\prime}(T), \partial f(T) / \partial x_{i j}, 1 \leq i, j \leq l+1\right) .
$$

To prove the assertion we must show that the codimension of this ideal in $S$ is at least three. If we add $T$ to $J$, by Krull's theorem, it is enough to show

\footnotetext{
${ }^{1}$ The authors have extended this result to all semisimple Lie algebras.
} 
that the codimension of $(J, T) / T$ is at least four. But this is the ideal of $R$ generated by the partial derivatives of the determinant of the generic matrix $x$, in other words, the determinantal ideal of the submaximal minors of $x$-which has height four by the classical formula (see [4]).

Finally we show that $\mathscr{C}=S$. More in fact will be proved: The embedding of $S$ into $\mathscr{L}$ splits in codimension less than three. Indeed, we can view $\mathscr{L}$ as an $S$-module (with multiplication of the left, say) and show that for each prime ideal $M$ of $S$, of codimension at most two, the localization $S_{M}$ splits off $\mathscr{L}_{M}$.

If we put $P=R \cap M$, observe that since $S$ is integral over $R$, the depth of $\mathscr{L}_{M}$ over $S_{M}$ equals the depth of $\mathscr{L}_{P}$ over $R_{P}$, that is $\mathscr{L}_{M}$ is a free $S_{M^{-}}$ module. In the exact sequence

$$
0 \rightarrow S_{M} \rightarrow \mathscr{L}_{M} \rightarrow H_{M} \rightarrow 0,
$$

we show that $H_{M}$ is a free $S_{M}$-module, in other words, $\operatorname{Tor}_{1}^{S}\left(H_{M}, k(M)\right)=0$, where $k(M)$ is the field of quotients of $S / M$. Tensoring the sequence with $k(M)$ yields

$$
\operatorname{Tor}_{1}^{S}(H, k(M)) \neq 0 \Leftrightarrow 1 \in M \mathscr{L}_{M} .
$$

In other words, there exists $s \in S \backslash M$ such that $s \in M \mathscr{L}$. This however, upon multiplication on the right by $\mathscr{L}$, yields $s \mathscr{L} \subset M \mathscr{L}$ and therefore $\mathscr{L}_{M}=M \mathscr{L}_{M}$, contradicting Nakayama's lemma.

2. Algebras of type $B_{l} . L$ is the algebra of skew-symmetric matrices of order $2 l+1 . \mathscr{C}_{0}$ will be the Lie subalgebra generated by odd powers of $x$; it is a free $R$-module of rank $l$.

To prove the assertion it is enough to verify that the ideal $I$ generated by the $l \times l$-minors of the embedding $\mathscr{C}_{0} \subset \mathscr{L}$, after some specialization, has codimension at least two, that is, it is not contained in any principal ideal. The specialization we choose places 0 's everywhere in the generic element $x$, except near the main diagonal:

$$
x^{*}=\left(\begin{array}{cccccc}
0 & t_{1} & 0 & \ldots & 0 & 0 \\
-t_{1} & 0 & t_{2} & \ldots & 0 & 0 \\
\vdots & \vdots & \vdots & \ddots & \vdots & \vdots \\
0 & 0 & 0 & \cdots & 0 & t_{2 l} \\
0 & 0 & 0 & \ldots & -t_{2 l} & 0
\end{array}\right) .
$$

The minimal polynomial of the matrix $x^{*}$ has degree $2 l$, even if we further specialize one of the $t_{i}$ to zero. This means that the specialization $I^{*}$ of the ideal $I$ is not divisible by any of the $t_{i}$. We may now invert all these variables.

To show that $I^{*}$ is the unit ideal, let us look at the generator given by the minor corresponding to the basis vectors $e_{i j}, j$ even, of $M_{2 l}\left(R^{*}\right)$. Thus $x^{*}$ has nonzero component $\left(=t_{1}\right)$ at $e_{12}$ and zeros in the rest of the top row. In general, $\left(x^{*}\right)^{2 i-1}$ has a nonzero component at $e_{1,2 i}$ and zeros beyond that 
position. This follows simply by looking at the connected path that must be used to find that entry in $\left(x^{*}\right)^{2 i-1}$. Furthermore, that component is a monomial in the $t_{i}$ 's. This suffices to prove the assertion.

3. Algebras of type $D_{l}$. The representation chosen for $L$ will be as the skewsymmetric matrices of order $2 l . \mathscr{C}_{0}$ is the Lie subalgebra of odd powers of $x$ up to order $2 l-3$, together with the canonical matrix $x^{\prime}$ made up of the Pfaffians of $x$ of order $2 l-2$; it is a free $R$-module of rank $l$.

The argument of the preceding case goes through up to assuming that $I^{*}$ is not divisible by any of the $t_{i}$ 's. The rest uses the fact that $\left(x^{*}\right)^{\prime}$ has a nonzero, monomial, entry at $e_{1,2 l}$.

4. Algebras of type $C_{l}$. The representation for the symplectic Lie algebra will be that as square $s l \times 2 l$-matrices (cf. [2, p. 200])

$$
\left(\begin{array}{cc}
A & B \\
C & -s A^{t} s
\end{array}\right)
$$

where $s$ is the $l \times l$-matrix with 1 's along the antidiagonal, and $A, B, C$ are matrices of order $l$ such that $B=s B^{t} s$ and $C=s C^{t} s$.

This time we define $\mathscr{C}_{0}$ to be the submodule generated by $x, x^{3}, \ldots$, $x^{2 l-1}$. It sits as a $R$-direct summand in the subalgebra $S=R[x]$ of $M_{2 l}(R)$. Because $\mathscr{L}$ itself is a direct summand of $M_{2 l}(R)$, it suffices to show that the embedding of $S$ into $M_{2 l}(R)$ splits in codimension at most one. To this end, we argue as in the case of $A_{l}$.

Take the Cayley-Hamilton's polynomial of the generic element $x$ of $L$ :

$$
f(T)=T^{2 l}+\sigma_{1} T^{2 l-1}+\cdots+(-1)^{2 l} \sigma_{2 l}, \quad \sigma_{2 l}=\operatorname{det}(x) .
$$

As for $A_{l}$ let us estimate the codimension of the Jacobian ideal of $f(T)$.

Lemma 5.3. $S$ is regular in codimension one.

Proof. It suffices to show that the ideal generated by the partial derivatives of $\Delta=\operatorname{det}(x)$, with respect the set of variables $x_{i j}$ 's, corresponding to the chosen representation, has codimension at least 3 .

Effect the following transformation on $x$ : Change the signs of its last $l$ columns and multiply the resulting matrix on the left by

$$
J=\left(\begin{array}{ll}
0 & s \\
s & 0
\end{array}\right)
$$

to obtain the symmetric matrix

$$
\left(\begin{array}{cc}
s C & A^{t} s \\
s A & -s B
\end{array}\right) .
$$

Its determinant differs from $\Delta$ at most in the sign. We thus must show that for a given generic, symmetric $n \times n$ matrix $X$, the ideal $J$ generated by the derivatives of its determinant has codimension at least 3. This is obvious if $X$ is a $2 \times 2$ matrix. Assume $n>2$ and that $P$ is a minimal prime of $J$ of codimension 2; one of the diagonal variables, say $x_{11}$, does not lie in $P$. 
The induction step is set up as follows. Passing over $R$ localized at the powers $x_{11}$, there exists a matrix $M$ such that

$$
M^{t} X M=\left(\begin{array}{cc}
x_{11} & 0 \\
0 & X^{\prime}
\end{array}\right) .
$$

with $X^{\prime}$ a symmetric matrix whose entries are

$$
x_{i j}^{\prime}=x_{i j}-x_{1 i} x_{1 j} / x_{11} \quad i, j \geq 2
$$

and $M$ has determinant 1 (see [13, p. 596] for full details).

5. Algebras of type $G_{2}$. For these algebras we have been unable to exhibit $\mathscr{C}$ except over fields of characteristic $p>0$ for which 2 is a quadratic residue. One nonetheless has

Proposition 5.4. The generic Cartan subalgebra of an algebra of type $G_{2}$ is a free $R$-module.

Proof. Let $x$ be a generic element of $L$. We claim that the module $R x$ splits off $\mathscr{C}$. Since $\mathscr{C}$ is a reflexive module of rank two, this will imply that it is $R$-free.

Consider the embeddings of $R x$ into both $\mathscr{L}$ and $\mathscr{C}$, and the resulting commutative diagram provided by the snake lemma:

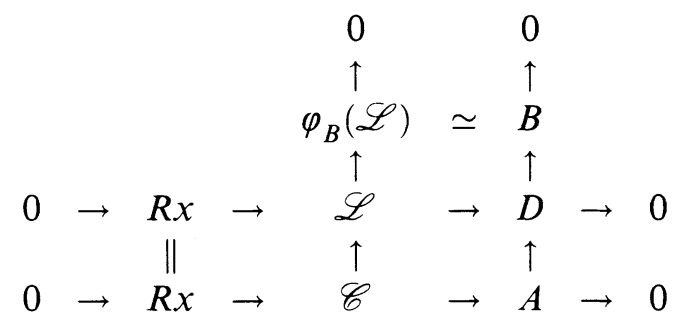

$D$ is a module of projective dimension one that is free in codimension at least two; it is thus reflexive (see [20, Proposition 3]). But $B$ is a torsion-free module so that $A$ is a rank one reflexive module and therefore free since $R$ is factorial.

For the algebras above therefore the Jacobian module is torsion-free by Theorem 2.5. It is comparable to the assertion that in a semisimple Lie algebra, the set of elements $a$ whose centralizer has dimension $l=\operatorname{rank}(L)$ is not contained in the complement of a hypersurface. Another consequence is that the ideal $J(L)$ contains all the quadrics and cubics of its radical.

Calculations using Macaulay showed that the commuting varieties of the classical Lie algebras, for $l \leq 2$, are Cohen-Macaulay domains. On the other hand, since the projective dimension of the Jacobian module is two (outside of trivial cases), $\mathscr{C}(L)$ cannot be factorial (cf. [9, Proposition 7.2]).

\section{REFERENCES}

1. L. Avramov, Complete intersections and symmetric algebras, J. Algebra 73 (1980), 249-280.

2. N. Bourbaki, Groupes et algèbres de Lie, Chapitres VII-VIII, Hermann, Paris, 1975. 
3. D. Buchsbaum and D. Eisenbud, What makes a complex exact? J. Algebra 25 (1973), 259-268.

4. J. Eagon and D. G. Northcott, Ideals defined by matrices and a certain complex associated with them, Proc. Roy. Soc. London 269 (1962), 188-204.

5. F. R. Gantmacher, Applications of the theory of matrices, Interscience, New York, 1959.

6. M. Gerstenhaber, On dominance and varieties of commuting matrices, Ann. of Math. 73 (1961), 324-348.

7. R. M. Guralnick, A note on pairs of matrices with rank one commutators, Linear and Multilinear Algebra 8 (1979), 97-99.

8. K. Hulek, A remark on certain matrix varieties, Linear and Multilinear Algebra 10 (1981), 169-172.

9. J. Herzog, A. Simis and W. V. Vasconcelos, On the arithmetic and homology of algebras of linear type, Trans. Amer. Math. Soc. 283 (1984), 661-683.

10. C. Huneke, On the symmetric algebra of a module, J. Algebra 69 (1981), 113-119.

11. C. Huneke and M. E. Rossi, The dimension and components of symmetric algebras, J. Algebra 98 (1986), 200-210.

12. N. Jacobson, Lie algebras, Interscience, New York, 1962.

13. T. Józefiak, Ideals generated by the minors of a symmetric matrix, Comment. Math. Helv. 53 (1978), 595-607.

14. H. Kleppe and D. Laksov, The algebraic structure and deformation of Pfaffian schemes, $\mathbf{J}$. Algebra 64 (1980), 167-189.

15. H. Matsumura, Commutative ring theory, Cambridge Univ. Press, 1986.

16. T. Motzkin and O. Taussky, Pairs of matrices with property L. II, Trans. Amer. Math. Soc. 80 (1955), 387-401.

17. M. Nagata, Local rings, Interscience, New York, 1962.

18. M. G. Neubauer, The variety of pairs of matrices with $\operatorname{rank}(A B-B A) \leq 1$, Proc. Amer. Math. Soc. 105 (1989), 787-792.

19. R. W. Richardson, Commuting varieties of semisimple Lie algebras and algebraic groups, Compositio Math. 38 (1979), 311-327.

20. P. Samuel, Anneaux gradués factoriels et modules réflexifs, Bull. Soc. Math. France 92 (1964), 237-249.

21. A. Simis and W. V. Vasconcelos, On the dimension and integrality of symmetric algebras, Math. Z. 177 (1981), 341-358.

22. __ Krull dimension and integrality of symmetric algebras, Manuscripta Math. 61 (1988), 63-78.

Department of Mathematics, University of North Dakota, Fargo, North Dakota 58105 (Current address Of J. P. Brennan)

Department of Mathematics, Rutgers University, New Brunswick, New Jersey 08903 (Current address of M. V. Pinto and W. V. Vasconcelos) 